\title{
Exploiting Metallophilicity for the Assembly of Inorganic Nanocrystals and Conjugated Organic Molecules
}

\author{
Mariona Dalmases, ${ }^{[a, b]}$ Elisabet Aguiló, ${ }^{[a]}$ Jordi Llorca, ${ }^{[c]}$ Laura Rodríguez, $^{*[a]}$ and \\ Albert Figuerola*[a, b]
}

The accurate engineering of interfaces between inorganic nanocrystals and semiconducting organic molecules is currently viewed as key for further developments in critical fields such as photovoltaics and photocatalysis. In this work, a new and unconventional source of interface interaction based on metal-metal bonds is presented. With this aim, an Au' organometallic gelator was exploited for the formation of hydrogel- like nanocomposites containing inorganic nanoparticles and conjugated organic molecules. Noteworthy, the establishment of metallophilic interactions at the interface between the two moieties greatly enhances interparticle coupling in the composites. Thus, we believe that this new hybrid system might represent a promising alternative in several fields, such as in the fabrication of improved light-harvesting devices.

\section{Introduction}

Plasmonic nanostructures have recently gained a lot of importance as both strong visible-light absorbers and exciton-dissociation enhancers in photovoltaic and photocatalytic devices. $^{[1,2]}$ Indeed, in many proposed plasmon-enhanced light-harvesting systems, the active material layer is formed by a blend of organic and inorganic domains, namely, polymers or small conjugated organic molecules and noble metal nanoparticles (NPs) or metallic contacts. ${ }^{[3-6]}$ Thus, the study of interfaces between materials has become an issue of paramount importance in the field, and they must be accurately engineered to ease charge-carrier percolation through the composite and consequently maximize energy conversion or photocatalytic activity. ${ }^{[7-9]}$

An interesting approach to control the organization of an organic material is the use of noncovalent, supramolecular interactions: it is well known that semiconducting polymers and $\pi$ conjugated organic molecules can self-assemble to form functional gel-like structures based on $\pi-\pi$ stacking that lead to superior electronic properties. ${ }^{[10,11]}$ Inorganic NPs might then

[a] M. Dalmases, E. Aguiló, Dr. L. Rodríguez, Dr. A. Figuerola

Departament de Química Inorgànica

Universitat de Barcelona

Martí i Franquès, 1-11, 08028 Barcelona (Spain)

E-mail: laura.rodriguez@qi.ub.es

albert.figuerola@qi.ub.es

[b] M. Dalmases, Dr. A. Figuerola

Institut de Nanociència i Nanotecnologia (IN²UB)

Universitat de Barcelona

Martí i Franquès, 1-11, 08028 Barcelona (Spain)

[c] Dr. J. Llorca

Institut de Tècniques Energètiques i Centre de Recerca en NanoEnginyeria Universitat Politècnica de Catalunya Diagonal, 647, 08028 Barcelona (Spain)

$\square$ Supporting Information and the ORCID identification number(s) for the

(iD author(s) of this article can be found under http://dx.doi.org/10.1002/ cphc. 201600239

be embedded in these supramolecular systems. ${ }^{[12]}$ If polymeric or small organic molecule gelators are used for this purpose, the organic-inorganic interface is usually based on covalent bonds, ${ }^{[13,14]}$ electrostatic interactions, ${ }^{[15]}$ and/or hydrogen bonding. ${ }^{[16]}$

The use of metallogelators instead of organic-only gelators for the formation of either hydrogels or organogels has recently become the subject of study. ${ }^{[17,18]}$ Metallogelators are defined as low molecular weight gelators formed by the coordination of organic ligands to metallic ions. As in the case of $\pi$ conjugated organic gelators, metallogelators spontaneously self-assemble through a combination of noncovalent interactions, which results in the formation of filaments or aggregated structures. Noteworthy, the presence of a metal ion offers the possibility to establish, in some cases, metallophilic intermolecular interactions of strength comparable to that of the hydrogen bond, and this is the driving force for the formation of supramolecular structures. ${ }^{[19]}$ Whereas metal-metal bonds are widely observed in solid-state molecule-based crystalline systems, such a source of interaction has only recently been observed in solution, ${ }^{[20-23]}$ and to the best of our knowledge, it has never been explored as a way of anchoring functional molecules to nanostructured materials to enhance synergetic phenomena.

We present herein a new hydrosoluble nanocomposite (NCP) originating from the spontaneous supramolecular assembly of noble metal NPs and a highly fluorescent low molecular weight $\mathrm{Au}^{\prime}$ metallogelator. ${ }^{[24]}$ These novel hybrids are characterized by intimate organometallic-inorganic interfaces that induce a strong electronic coupling between the two components, and in this way, they become potential candidates for future optoelectronic devices or photocatalytic systems. Additionally, the method was successfully extended to inorganic NPs other than noble metals, which confirmed the large versatility of the strategy presented. 


\section{Results and Discussion}

The assembly of the two components leading to the formation of the hydrosoluble NCP was achieved simply by mixing two chloroform solutions at room temperature, separately containing oleylamine-capped Au NPs (Au-OLAm NPs) and the luminescent gold(I) alkynyl phosphine complex [Au(4-pyridylethynyl)(DAPTA)] (Figure 1a), for which DAPTA is 3,7-diacetyl-1,3,7triaza-5-phosphabicyclo[3.3.1]nonane, hereafter called complex 1 for simplicity. The precipitate obtained was easily separated by centrifugation and was washed with chloroform, and the solid was redispersed in a polar solvents such as water, methanol, or ethanol to form an optically clear blue solution, as shown in Figure $1 \mathrm{~b}$. Whereas transmission electron microscopy (TEM) images confirmed that the original size and shape of the hydrophobic Au-OLAm NPs were preserved after the formation of the hydrophilic NCP (see Figure S1 in the Supporting Information), field-emission scanning electron microscopy (FESEM) showed the formation of aggregates of metallic particles surrounded by a light contrasted shell of lower atomic weight, as
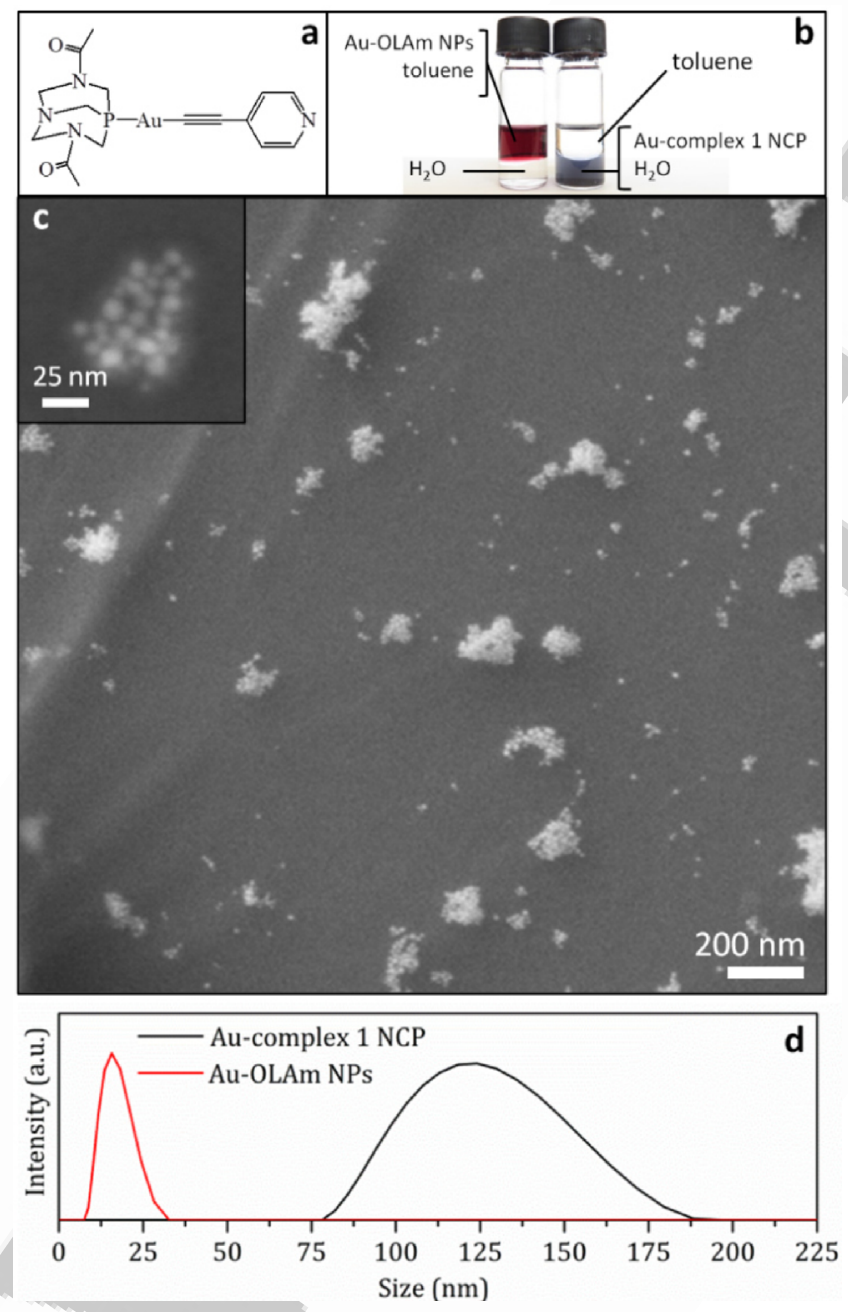

Figure 1. a) Molecular structure of complex 1. b) Solubility of the initial hydrophobic Au-OLAm NPs and the final hydrophilic Au-complex 1 NCP. c) FESEM micrograph of Au-complex 1 NCP. d) DLS measurements of hydrophobic Au-OLAm NPs and the hydrophilic Au-complex 1 NCP. shown in Figure 1c and inset, which suggested the presence of an organic coverage. Dynamic light scattering (DLS) measurements indicated that the solution was formed by a polydisperse distribution of colloidally stable aggregates of sizes ranging from 75 to $200 \mathrm{~nm}$, as indicated in Figure $1 \mathrm{~d}$. Although a minimum complex 1/Au NP molar ratio of 15000 was experimentally found to be necessary in the reaction medium to guarantee quantitative transfer of the NPs into water, no significant differences in the product obtained were observed for ratios ranging from approximately 20000 to 100000 , besides changes in the size dispersion of the aggregates formed, which could not be unequivocally linked to the varying ratio (see Figure S2). The DLS measurements also revealed that the aggregates were stable in solution for long periods of time, that is, up to 12 months at least, at room temperature as well as at $50^{\circ} \mathrm{C}$, and at any value of $\mathrm{pH}$ within the range between 4 and 9 (Figures S3 and S4).

The infrared (IR) spectra of the initial hydrophobic Au-OLAm NPs, free complex 1, and the synthesized hydrophilic NCP sample were recorded in $\mathrm{KBr}$ pellets, and they are shown in Figure $2 \mathrm{a}$. The NCP was washed prior to the experiments with dichloromethane to discard any unbound molecules of complex 1. The intense carboxylic $\mathrm{C}=\mathrm{O}$ and pyridinic $\mathrm{C}=\mathrm{N}$ stretching bands between $\tilde{v}=1500$ and $1700 \mathrm{~cm}^{-1}$ indicate the presence of molecules of complex 1 in the sample. Slight shifts to lower wavenumbers and the wider range of frequencies for the transitions observed, relative to the spectrum of free complex 1, suggest the effective chemisorption of the molecules on the surface of the metallic nanostructures. ${ }^{[25]}$ At the same time, this indicates that the molecules of complex 1 interact differently with the NPs, in agreement with the formation of a significantly thick envelope that confers solubility to the aggregates. Notably, the intensity of the aliphatic $\mathrm{C}-\mathrm{H}$ stretching bands at around $\tilde{v}=2900 \mathrm{~cm}^{-1}$ observed for the initial oleylamine-capped Au NPs suffers drastic damping, which evidences significant removal of the hydrophobic surfactant. The mass spectra of the sample show several characteristic peaks corresponding to free complex 1 with additional atoms of $\mathrm{Au}$; the latter is most probably knocked out from the metallic surface during the ionization process (see Figure S5). X-ray photoelectron spectroscopy (XPS) was also used for chemical characterization of the NCP. The XPS spectrum shown in Figure $2 b$ proves the coexistence of $\mathrm{Au}^{0}$ and $\mathrm{Au}^{\prime}$ species in the sample, in agreement with the formation of hybrid systems containing both metallic Au NPs and an Au'-based metallogelator. Notably, the XPS spectrum of the initial Au-OLAm NPs shows a signal that belongs exclusively to $\mathrm{Au}^{0}$, as expected from the absence of complex 1 in the sample (see Figure S6). Fitting of the XPS spectrum in the $N$ and $P$ regions indicates a N/P ratio of 3.6 in the NCP, in fair agreement with the theoretical ratio of 4 for complex 1, which further confirms its presence in the sample and the removal of the initial aliphatic amine surfactant (see Figure S7).

The UV/Vis spectra of the hybrid system at different $\mathrm{pH}$ values present two signals, as shown in Figure 3 . On the one side, there is a vibronically resolved band at $\lambda \approx 260 \mathrm{~nm}$ attributed to the metallogelator chromophore of complex 1 , which 


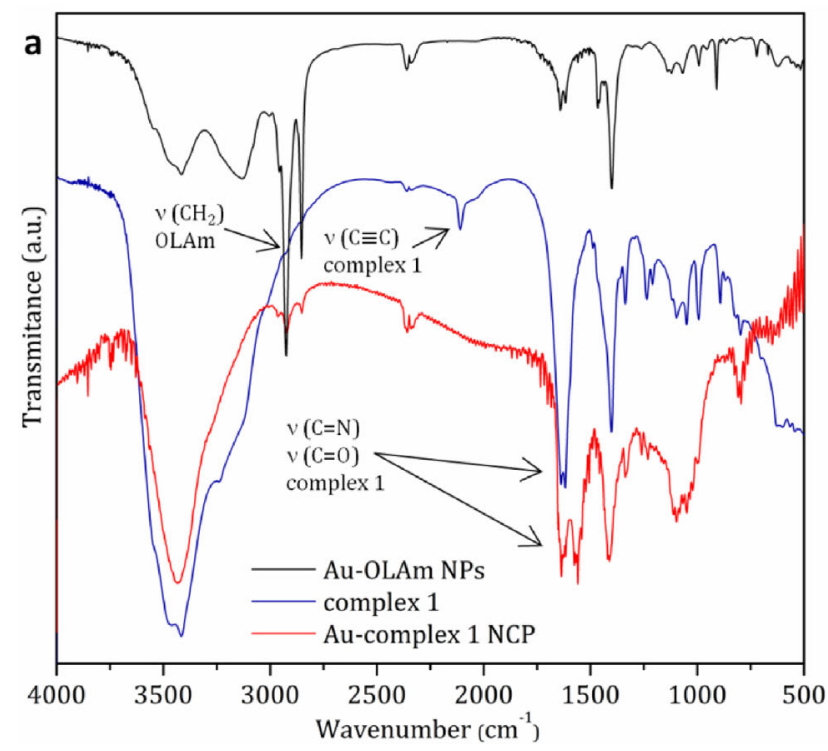

b

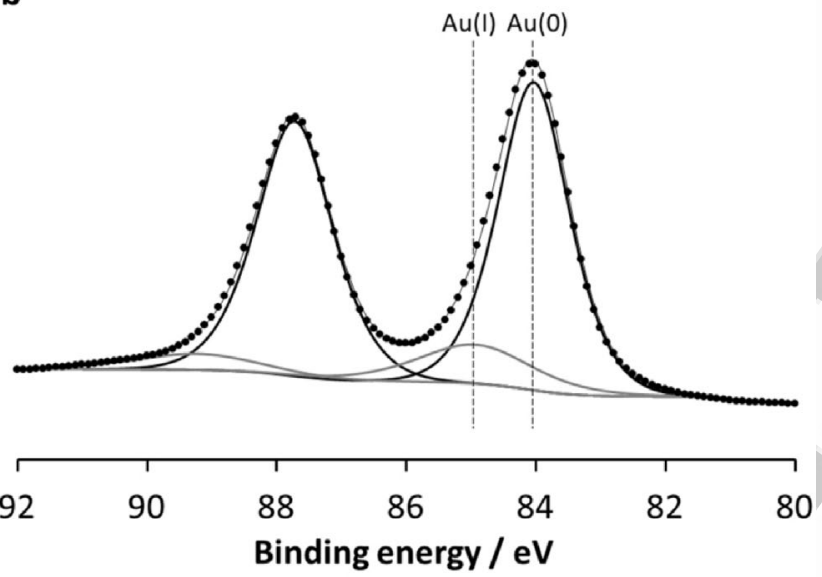

Figure 2. a) IR spectra of Au-OLAm NPs, free complex 1, and Au-complex 1 NCP. b) XPS spectrum of the Au-complex 1 NCP.

in its free form presents two characteristic bands at around this wavelength. ${ }^{[2]}$ On the other side, the signal at $\lambda=500-$ $600 \mathrm{~nm}$ is assigned to the characteristic surface plasmon resonance (SPR) band of gold NPs. To better study the formation of the NCP, two series of absorption spectra were recorded by fixing one of the components (complex 1 or Au-OLAm NPs) and by adding small amounts of the other successively at $\mathrm{pH}$ 7. Both experiments (see Figure S8) show a gradual shift and widening of the SPR band. The redshift and broadening of this band, relative to the band observed for the isolated oleylamine-capped Au NPs in toluene, denote agglomeration of the NPs. ${ }^{[26]}$ The collected data so far suggest that aggregation is due to trapping of the NPs within the entangled complex 1based structures. Our previous studies showed how aqueous solutions of free complex 1 spontaneously form hydrogel structures propagated through supramolecular interactions. ${ }^{[24]}$ Similar hydrogel structures are also likely formed in the presence of Au NPs, and they wrap around the NPs and guarantee their water solubility.

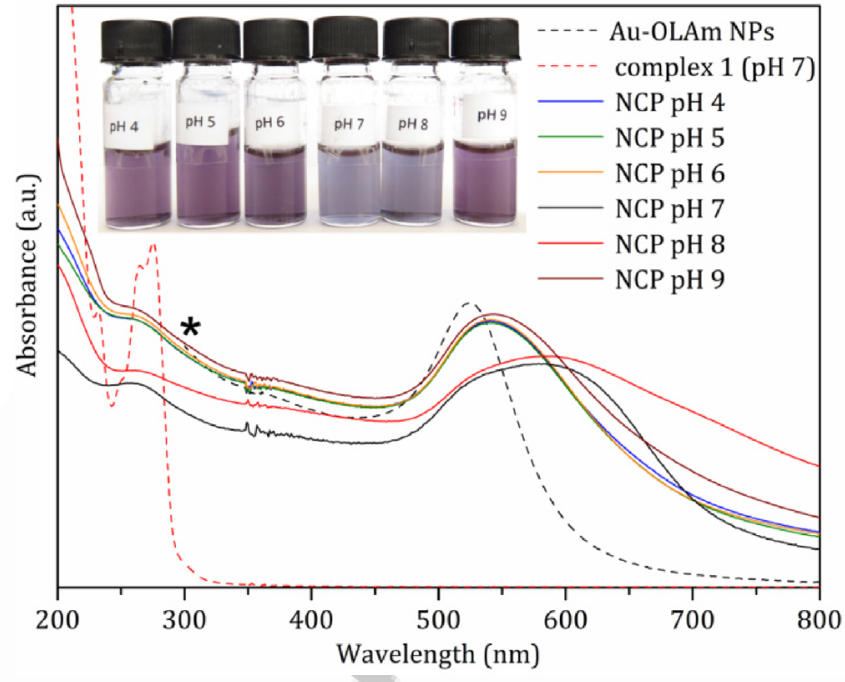

Figure 3. UV/Vis absorption spectra of hydrophilic the Au-complex $1 \mathrm{NCP}$ at different $\mathrm{pH}$ values, hydrophobic Au-OLAm NPs in toluene, and free complex 1 at neutral $\mathrm{pH}$. *For ease of understanding, the spectrum of Au-OLAm NPs in toluene is shown only above $\lambda=300 \mathrm{~nm}$, which avoids the appearance of the absorption of the solvent in the figure. Inset) Au-complex 1 NCP water dispersions at different $\mathrm{pH}$ values.

hydrogels 1 year after their synthesis was confirmed by DLS (Figure S4) and UV/Vis absorption spectroscopy: the redshifting and broadening of the SPR band are either retained or even enhanced relative to the fresh samples after such a long storage time, as shown in Figure S9. This long-term stability is most probably due to incorporation of a large amount of solvent molecules within the aggregates, typical of the hydrogellike behavior of the system.

As depicted in the inset of Figure 3 and in Figure S10, a reversible change in the color of the solution is observed from violet at low $(<7)$ and high $(>8) \mathrm{pH}$ to blue at neutral and slightly basic $\mathrm{pH}$. This translates into the largest redshift and broadening of the SPR band in neutral and slightly basic media ( $\mathrm{pH} 7-8$ ). However, the DLS measurements show no apparent shrinkage or variation in the size or shape of the aggregates at this $\mathrm{pH}$ (Figure S4). Consequently, the observed pHdependent optical properties are most likely linked to a subtle enhancement in interparticle interactions by strengthening specific chemical bonds than to an enhancement in interparticle interactions induced by large conformational or morphological changes of the aggregates. ${ }^{[15,27]}$ Interestingly, in the UV/ Vis spectra of water solutions of free complex 1 (without NPs) at $\mathrm{pH} 7-8$ (see Figure S11) there is notable intensification of the broad and weak band at $\lambda=300 \mathrm{~nm}$, assigned to aurophilic interactions, ${ }^{[28]}$ which indicates better capacity of complex 1 to create $\mathrm{Au}^{\prime}-\mathrm{Au}^{\prime}$ bonds at this $\mathrm{pH}$. Indeed, for the NCP sample, the ratio of absorption intensities at $\lambda=300$ and $260 \mathrm{~nm}\left(A_{300} / A_{260}\right)$ is maximum at $\mathrm{pH} 7-8$, and this confirms the presence of a higher amount of aurophilic interactions under these specific conditions (Figure S12). This fact, coinciding with the maximum enlargement in the SPR band at the same $\mathrm{pH}$, strongly suggests that metallophilicity plays a key role in interparticle plasmon coupling. 
All in all, these data confirm the formation of hydrosoluble Au-complex $1 \mathrm{NCPs}$ and illustrate how aurophilic interactions strengthen the interface between the inorganic NPs and the organometallic gelator and enhance the interparticle SPR coupling. To further confirm these findings, additional experiments were made by replacing the Au NPs by other types of inorganic nanocrystals of similar sizes but with a different chemical nature. $\mathrm{Ag} \mathrm{NPs}$ as well as $\mathrm{CdSe}$ quantum dots and $\mathrm{Fe}_{3} \mathrm{O}_{4}$, $\mathrm{Ag}_{2} \mathrm{Se}$, and $\mathrm{Ag}_{3} \mathrm{AuSe}_{2}$ nanocrystals were chosen as representative systems. They were separately introduced into a solution of complex 1 in chloroform and left to react by following exactly the same procedure as that described in the Experimental Section for the Au NPs. The Ag NPs behaved in a way analogous to that of the Au NPs. The formation of colloidally stable aggregates similar to that found in the Au-complex 1 NCP was confirmed by TEM, FESEM, and DLS measurements (see Figures $4 \mathrm{a}$ and S13-S15) although their size distribution was larger and around $200 \mathrm{~nm}$. IR spectroscopy and XPS (Figures S16 and S17) demonstrate the substitution of the initial surfactant (i.e., OLAm) by the $\mathrm{Au}^{\prime}$ organometallic complex. The broad SPR band with two shoulders observed in the visible absorption spectrum (Figure S18) is additional clear evidence of the coupling between the two components in the Ag-complex $1 \mathrm{NCP}$. Metallophilic $\mathrm{Ag}-\mathrm{Au}$ interactions are expected to play an important role in this system as they do in many supramolecular assemblies of organometallic molecules. ${ }^{[29]}$

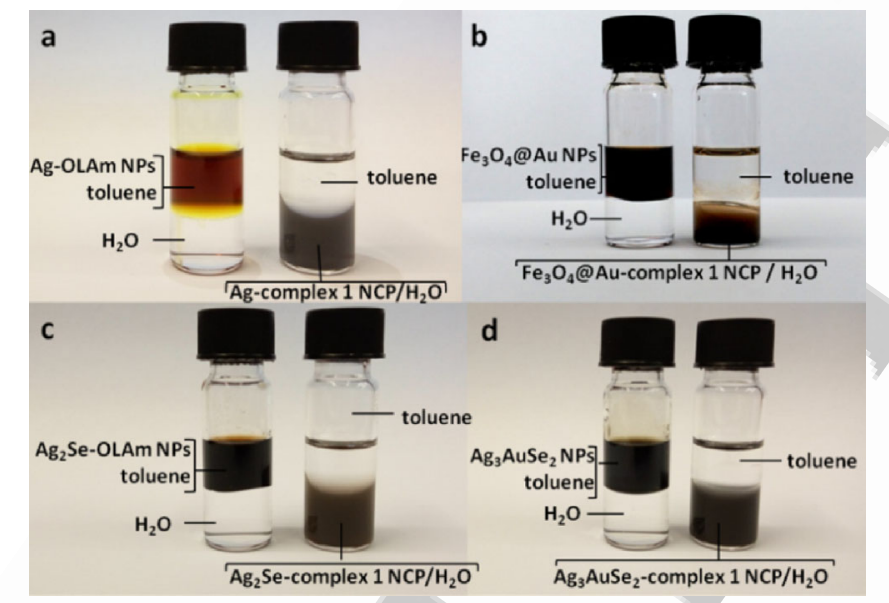

Figure 4. Solubility of the initial hydrophobic inorganic nanocrystals and the final hydrophilic NCPs of these nanocrystals with complex 1 .

On the contrary, no hydrophilic materials were obtained upon replacing the Au NPs by $\mathrm{CdSe}$ or $\mathrm{Fe}_{3} \mathrm{O}_{4}$ nanocrystals. This behavior is believed to be due to the absolute lack of affinity between the surface of these inorganic NPs and complex 1, most probably related to their inability to establish metallophilic interactions. However, a fine atomic Au coating of the surface of the $\mathrm{Fe}_{3} \mathrm{O}_{4}$ nanocrystals or the substitution of $\mathrm{Cd}^{\prime \prime}$ by $\mathrm{Ag}^{\prime}$ and/or $\mathrm{Au}^{\prime}$ in the chalcogenide material was sufficient to improve their affinity for complex 1 and to obtain stable aqueous solutions of $\mathrm{Fe}_{3} \mathrm{O}_{4} @ \mathrm{Au}$-complex $1 \mathrm{NCPs}, \mathrm{Ag}_{2} \mathrm{Se}$-complex
$1 \mathrm{NCPs}$, and $\mathrm{Ag}_{3} \mathrm{AuSe}_{2}$-complex 1 NCPs (see Figure $4 \mathrm{~b}-\mathrm{d}$ and Figure S19).

The strong fluorescence of free complex 1 impedes observation of its Raman scattering spectrum. However, the fluorescence of the metallogelator is quantitatively quenched in all the synthesized NCP samples as a consequence of efficient coupling between the inorganic NPs and the organometallic molecules. ${ }^{[30,31]}$ Moreover, molecules of complex 1 bound onto and placed in the proximity of plasmonic NPs experience surface-enhanced Raman scattering (SERS) processes that allow their vibrations to be observed, regardless of the low concentrations of the molecule in the NCP samples $(<0.01 \%$ in weight) ${ }^{[32]}$ Thus, the study of complex 1 in both Au- and Agbased NCP systems by Raman spectroscopy was performed, and the corresponding spectra are shown in Figure 5. Strong bands are observed at Raman shifts of about $1650-1600 \mathrm{~cm}^{-1}$, which can be assigned to the carbonyl and $\mathrm{C}=\mathrm{N}$ vibrations of the organometallic complex, as well as at $1200 \mathrm{~cm}^{-1}$, assigned to $\mathrm{C}-\mathrm{N}$ vibrations, and at $1000 \mathrm{~cm}^{-1}$, assigned to $\mathrm{C}-\mathrm{P}$ vibrations. It was reported that SERS processes are stronger for $\mathrm{Ag}$ NPs than for Au NPs. ${ }^{[33]}$ In fact, the SERS spectrum of the Agcomplex $1 \mathrm{NCP}$ (Figure 5 bottom) shows Raman scattering intensities around two orders of magnitude higher than those observed for the Au analogue at the same concentration and under the same measuring conditions. Remarkably, the Raman spectrum of the Ag-complex $1 \mathrm{NCP}$ exhibits a broad and strong band at a Raman shift of about $2000-2100 \mathrm{~cm}^{-1}$, assigned to the $C \equiv C$ vibration of complex 1 , which further evidences the presence of the metallogelator in the NCP.

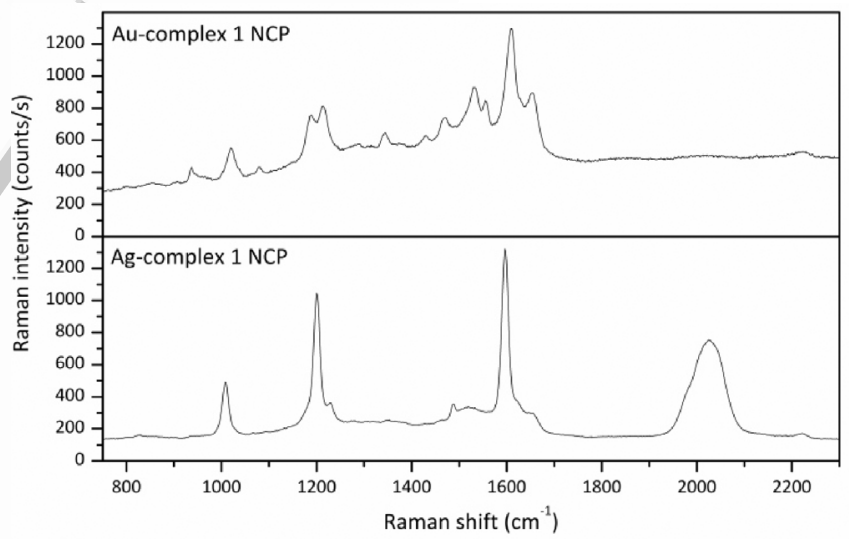

Figure 5. Raman spectra of the top) Au-complex $1 \mathrm{NCP}(\times 100)$ and bottom) Ag-complex 1 NCP.

\section{Conclusions}

To conclude, a new strategy was presented for the functionalization and coupling of noble metal, magnetic, and semiconductor NPs with conjugated organic moieties by means of direct metal-metal bonds. With this purpose, a small-moleculeconjugated metallogelator was used, which was characterized by the presence of a Au' ion covalently linked to a short conjugated $\pi$ system, with the ability to establish strong metallo- 
philic interactions with the surface atoms of inorganic NPs. Such a new source of chemical affinity induced the room-temperature formation of stable NCPs that were soluble in water and alcohol solutions for long periods of time. We believe that this type of system could represent a significant improvement in terms of interface design between functional inorganic NPs and photoactive polymers, as metallogelators are prone to create a direct link between surface atoms of nanostructures and $\pi-\pi$ stacking systems in semiconducting polymers, which chemically smooths their interface. By way of example, significant improvements in the field of plasmon-enhanced light-harvesting devices are expected thereof. The potential of other organometallic gelators for the successful functionalization of inorganic NPs, as well as the capacity of these novel NCPs to form functional blends of materials, are currently being addressed.

\section{Experimental Section}

\section{Chemicals}

Gold(III) chloride trihydrate $\left(\mathrm{HAuCl}_{4} \cdot 3 \mathrm{H}_{2} \mathrm{O}, \geq 99.9 \%\right)$, silver nitrate $\left(\mathrm{AgNO}_{3}, \geq 99 \%\right)$, iron(III) chloride $\left(\mathrm{FeCl}_{3}, 97 \%\right)$, tetraoctylammonium bromide (TOAB, 98\%), oleylamine (OLAm, 70\%), oleic acid (OLAc, $\geq 99 \%)$, 1-octadecene (ODE, 90\%), methanol ( $\mathrm{MeOH}$, $\geq 99.6 \%$ ), and toluene $(99.9 \%)$ were purchased from Sigma-Aldrich. Cadmium oxide (CdO, 99\%), selenium powder (Se, 99.99\%), silver chloride $(\mathrm{AgCl}, 99.9 \%)$, tri-n-octylphosphine oxide (TOPO, 99\%), and tri-n-octylphosphine (TOP, 97\%) were obtained from Strem Chemicals. Octadecylphosphonic acid (ODPA, 99\%) was purchased from Polycarbon Industries. Sodium oleate $(\mathrm{NaOL})$ was obtained from Tokyo Chemical Industry (TCI). 2-Propanol (iPrOH, for HPLC) and chloroform $\left(\mathrm{CHCl}_{3}, 99.2 \%\right)$ were purchased from VWR Chemicals. Absolute ethanol (99.5\%), ethanol (EtOH, $96 \% \mathrm{v} / \mathrm{v})$, and hexane $(95 \%)$ were obtained from Panreac. All reagents and solvents were used without further purification.

\section{Syntheses}

[Au( $\left.\left.\mathrm{C} \equiv \mathrm{C}-\mathrm{C}_{5} \mathrm{H}_{4} \mathrm{~N}\right)(\mathrm{DAPTA})\right]$ (complex 1): Complex 1 was synthesized according to previously reported methodology. ${ }^{[2]}$ Briefly, solid DAPTA was added to a suspension of $[\mathrm{Au}(\mathrm{C} \equiv \mathrm{Cpy})]_{n}$ (previously synthesized ${ }^{[34]}$ in $\mathrm{CH}_{2} \mathrm{Cl}_{2}$. After $45 \mathrm{~min}$ of stirring at room temperature, the resulting pale-yellow solution was concentrated, and hexane was added to precipitate a pale-yellow solid in quantitative yield.

Au NPs (Au-OLAm NPs): The synthesis of gold NPs was adapted from that described by $\mathrm{Yu}$ and co-workers ${ }^{[35]}$ as follows: A mixture of ODE $(30 \mathrm{~mL})$, OLAC $(4.5 \mathrm{~mL})$, and OLAm $(5.5 \mathrm{~mL})$ was stirred in a $100 \mathrm{~mL}$, three-necked flask for $20 \mathrm{~min}$ at $120^{\circ} \mathrm{C}$ under a $\mathrm{N}_{2}$ atmosphere. Meanwhile, in a glovebox $\mathrm{HAuCl}_{4} \cdot 3 \mathrm{H}_{2} \mathrm{O}(120 \mathrm{mg}$, $0.30 \mathrm{mmol})$ was dissolved in ODE $(7.5 \mathrm{~mL})$ and OLAm $(1.5 \mathrm{~mL})$ in an auxiliary vial. Once the purge was complete, the solution of the gold precursor was injected into the flask and the temperature was fixed at $150^{\circ} \mathrm{C}$. After $30 \mathrm{~min}$, the heating was stopped, and the solution was washed with 2 equivalent volumes of $i \mathrm{PrOH}(2 \times)$, and centrifuged at $4500 \mathrm{rpm}$ for $4 \mathrm{~min}$. The product was redispersed in toluene.

Ag NPs (Ag-OLAm NPs): The synthesis of the silver NPs was based on that reported by Shen and co-workers. ${ }^{[36]}$ Briefly, in a $100 \mathrm{~mL}$, three-necked flask $\mathrm{AgNO}_{3}(85 \mathrm{mg}, 0.50 \mathrm{mmol})$ and OLAm (2 mL) were dissolved in toluene $(50 \mathrm{~mL})$. Under nitrogen flow, the solution was heated to $110^{\circ} \mathrm{C}$ and kept at this temperature for $6 \mathrm{~h}$. After this time, the mixture was cooled down naturally to room temperature, washed with $\mathrm{EtOH}(50 \mathrm{~mL}, 1 \times)$, and centrifuged for $5 \mathrm{~min}$ at $6000 \mathrm{rpm}$. The precipitate was redispersed in toluene.

$\mathrm{Fe}_{3} \mathrm{O}_{4}$ NPs: The synthesis of the iron oxide NPs was based on that described by Park and co-workers. ${ }^{[37]}$ Under a $\mathrm{N}_{2}$ atmosphere, a mixture of iron oleate solution $(5 \mathrm{~mL}, 1.00 \mathrm{mmol}), \mathrm{NaOL}(76 \mathrm{mg}$, $0.25 \mathrm{mmol})$, OLAc $(88.5 \mu \mathrm{L}, 0.25 \mathrm{mmol})$, and ODE $(10 \mathrm{~mL})$ was heated for $1 \mathrm{~h}$ at $70^{\circ} \mathrm{C}$. Immediately thereafter, the temperature was raised to $100^{\circ} \mathrm{C}$, and the mixture was heated at a rate of $3^{\circ} \mathrm{C} \mathrm{min}{ }^{-1}$ to $318-320^{\circ} \mathrm{C}$ and kept at this temperature for $30 \mathrm{~min}$. Heating was then stopped, and the crude solution was cooled to room temperature in air and left to stir for an additional $1 \mathrm{~h}$. The final solution was washed with $\mathrm{PrOH}(3 \times)$, centrifuged for $4 \mathrm{~min}$ at $4000 \mathrm{rpm}$, and redispersed in toluene. The iron oleate used as a precursor was prepared previously with $\mathrm{FeCl}_{3}$ and $\mathrm{NaOL}$.

CdSe NPs: The synthesis of the cadmium selenide NPs was performed by using the method published by Carbone and co-workers. ${ }^{[38]}$ In a $25 \mathrm{~mL}$, three-necked flask under continuous vacuum and stirring TOPO $(3.0 \mathrm{~g})$, ODPA $(280 \mathrm{mg}, 0.84 \mathrm{mmol})$, and CdO $\left(60 \mathrm{mg}, 0.47 \mathrm{mmol}\right.$ ) were heated at $150^{\circ} \mathrm{C}$ for $2 \mathrm{~h}$. Under a $\mathrm{N}_{2}$ atmosphere, the temperature was then raised to $370^{\circ} \mathrm{C}$. Once at $300^{\circ} \mathrm{C}$, TOP $(150 \mathrm{~g}, 4 \mathrm{mmol})$ was injected into the solution. At $370^{\circ} \mathrm{C}$, a solution of Se $(58 \mathrm{mg}, 0.73 \mathrm{mmol})$ in TOP $(360 \mathrm{mg}$, $0.97 \mathrm{mmol}$ ) was injected. After $15 \mathrm{~s}$ of the last injection, the reaction was stopped by removing the heating mantle and letting the flask cool down naturally. At $100^{\circ} \mathrm{C}$, toluene $(5 \mathrm{~mL})$ was added to the solution to avoid solidification. Once at room temperature, the solution was washed with $\mathrm{MeOH}(3 \times)$ and redispersed in toluene.

Atomic gold coating of iron oxide NPs $\left(\mathrm{Fe}_{3} \mathrm{O}_{4} @ \mathrm{Au} \mathrm{NPs}\right)$ : Iron oxide NPs were gold coated by following a procedure published by Malyutin and co-workers. ${ }^{[39]}$ Briefly, a solution of $\mathrm{Fe}_{3} \mathrm{O}_{4} \mathrm{NPs}(4 \mathrm{mg})$, $\mathrm{CHCl}_{3}(1 \mathrm{~mL})$, and OLAm $(0.8 \mathrm{~mL})$ was stirred for $1 \mathrm{~h}$ at room temperature. Meanwhile, $\mathrm{HAuCl}_{4} \cdot 3 \mathrm{H}_{2} \mathrm{O}(98.5 \mathrm{mg}, 0.25 \mathrm{mmol})$ and OLAm $(0.2 \mathrm{~mL})$ were dissolved in $\mathrm{CHCl}_{3}(1 \mathrm{~mL})$. This solution was added dropwise to the NP solution and stirred for $24 \mathrm{~h}$. The final solution was purified with $\mathrm{EtOH}$ and then washed with a mixture of EtOH/hexane $(5: 1,2 \times)$. The final precipitate was redispersed in toluene.

$\mathrm{Ag}_{2} \mathrm{Se} \mathrm{NPs}$ : The synthesis of the $\mathrm{Ag}_{2} \mathrm{Se}$ NPs was adapted from that published by Sahu and co-workers. ${ }^{[40]}$ Briefly, TOPO $(7.8 \mathrm{~g})$ and OLAm $(6.6 \mathrm{~mL})$ were degassed under vacuum at $120^{\circ} \mathrm{C}$ for $30 \mathrm{~min}$. Meanwhile, two precursor solutions were prepared in a glovebox: Se $(474 \mathrm{mg}, 6 \mathrm{mmol})$ was dissolved in TOP $(6 \mathrm{~mL})$, and $\mathrm{AgCl}$ (572 mg, $4 \mathrm{mmol}$ ) was dissolved in TOP $(4 \mathrm{~mL})$. Under a $\mathrm{N}_{2}$ atmosphere, the temperature was raised to $180^{\circ} \mathrm{C}$ and the TOP-Se solution was injected. Once the temperature was recovered, the $\mathrm{AgCl}-$ TOP precursor solution was injected. After 20 min of reaction, the heating was stopped and the solution was left to cool naturally. Once at $50^{\circ} \mathrm{C}, \mathrm{BuOH}(5 \mathrm{~mL})$ was added to the reaction flask to avoid solidification of the solvent. Finally, the solution was washed with EtOH $(3 \times)$, centrifuged for $4 \mathrm{~min}$ at $4500 \mathrm{rpm}$, and redispersed in toluene $(4 \mathrm{~mL})$.

$\mathrm{Ag}_{3} \mathrm{AuSe}_{2} \mathrm{NPs}$ : A Ag $\mathrm{Ag}_{2} \mathrm{Se}$ NPs solution $(3.6 \mu \mathrm{M}, 180 \mu \mathrm{L})$ was mixed with a toluene $(2 \mathrm{~mL})$ solution of $6 \mathrm{~mm} \mathrm{Au}$ 'II-TOAB and shook for $1 \mathrm{~h}$. After this time, the final solution was washed with $\mathrm{EtOH}(1 \times)$ and redispersed in toluene. The $A u^{\prime \prime \prime}-$ TOAB solution was prepared by following the methodology published by Yang and co-workers. ${ }^{[41]} \mathrm{HAuCl}_{4} \cdot 3 \mathrm{H}_{2} \mathrm{O}(28.3 \mathrm{mg}, 70 \mu \mathrm{mol})$ was dissolved in deionized water $(12 \mathrm{~mL})$ and mixed with TOAB $(44 \mathrm{mg}, 80 \mu \mathrm{mol})$ dissolved in 
EtOH $(12 \mathrm{~mL})$. After $3 \mathrm{~min}$ of stirring, toluene $(12 \mathrm{~mL})$ was added to the solution and stirring was continued for $3 \mathrm{~min}$. Finally, the $\mathrm{Au}^{\prime \prime \prime}-$ TOAB toluene solution phase was separated.

Au-complex 1 NCP: The functionalization of Au NPs with complex 1 was performed by a reaction at room temperature in $\mathrm{CHCl}_{3}$. Complex $1(4.2 \mathrm{mg}, 8 \mu \mathrm{mol})$ was dissolved in $\mathrm{CHCl}_{3}(8 \mathrm{~mL})$ and mixed with Au NPs $(0.17 \mathrm{nmol})$. The solution was shook for $2 \mathrm{~h}$ and centrifuged for $3 \mathrm{~min}$ at $4500 \mathrm{rpm}$. The precipitate was redispersed in water.

The formation of NCPs of complex 1 with inorganic NPs other than $\mathrm{Au}$ (Ag NPs, $\mathrm{Fe}_{3} \mathrm{O}_{4} \mathrm{NPs}, \mathrm{CdSe} \mathrm{NPs}, \mathrm{Fe}_{3} \mathrm{O}_{4} @ \mathrm{Au} \mathrm{NPs}, \mathrm{Ag}_{2} \mathrm{Se} \mathrm{NPs}$, and $\mathrm{Ag}_{3} \mathrm{AuSe}_{2} \mathrm{NPs}$ ) was adapted from the protocol described above by using $\mathrm{Ag} / \mathrm{Fe}_{3} \mathrm{O}_{4} / \mathrm{CdSe} / \mathrm{Fe}_{3} \mathrm{O}_{4} @ \mathrm{Au} / \mathrm{Ag}_{2} \mathrm{Se} / \mathrm{Ag}_{3} \mathrm{AuSe}_{2} \mathrm{NPs}$ instead of $\mathrm{Au}$ NPs.

\section{Characterization methods}

Field-emission scanning electron microscopy (FESEM) images were recorded with a Zeiss Neon40 scanning electron microscope Crossbeam Station equipped with a field-emission electron source operating at $5 \mathrm{kV}$.

A Zetasizer NanoS Spectrometer was used for the dynamic light scattering (DLS) measurements. The samples were measured in quartz cuvettes.

Infrared (IR) spectra were recorded with a FTIR 520 Nicolet Spectrophotometer. For the measurements, a pellet of a mixture of the sample and $\mathrm{KBr}$ was used.

X-ray photoelectron spectroscopy (XPS) was performed with a SPECS system equipped with an Al anode XR50 source operating at $150 \mathrm{~mW}$ and a Phoibos 150 MCD-9 detector. The pass energy of the hemispherical analyzer was set at $25 \mathrm{eV}$, and the energy step was set at $0.1 \mathrm{eV}$. The binding energy (BE) values were referred to the $\mathrm{C} 1 \mathrm{~s}$ peak at $284.8 \mathrm{eV}$.

ESI $\square$ ok? $\square(+)$ mass spectra were recorded with a 4800Plus MALDI TOF/TOF Analyzer Applied Biosystems MDS SCIEX.

A Cary 100 Scan 388 Varian UV/Vis spectrophotometer and a NanoLog Horiba Jobin Yvon fluorometer were used with quartz cuvettes for optical characterization.

Raman spectrum were acquired with a Jobin-YvonLabRamHR 800 spectrophotometer coupled with an Olympus BXFM optical microscope by using a $\lambda=532 \mathrm{~nm}$ laser.

A Hitachi H800 MP conventional transmission electron microscope equipped with Bioscan Gatan camera and a tungsten filament operating at an acceleration voltage of $200 \mathrm{kV}$ and $3 \mu \mathrm{m}$ spot size was used for the morphological study of all synthesized nanostructures. The samples were prepared by drop casting and evaporation of the solvent onto carbon-coated $300 \mu \mathrm{m}$ mesh Cu grids.

The compositions and concentrations of the nanoparticles solutions were determined by inductively coupled plasma atomic emission spectroscopy (ICP-AES). The measurements were performed with an Optima 3200 RL PerkinElmer spectrometer. For the measurements, $50 \mu \mathrm{L}$ of the solutions was precipitated in $\mathrm{MeOH}$ and redispersed in $\mathrm{CHCl}_{3}$. The solution was evaporated in an oven overnight at $90^{\circ} \mathrm{C}$. Aqua regia $(2.5 \mathrm{~mL})$ and $\mathrm{H}_{2} \mathrm{O}_{2}(0.7 \mathrm{~mL})$ were added to the precipitate before the vial was sealed and then heated to $90^{\circ} \mathrm{C}$ for $72 \mathrm{~h}$. The resulting solution was transferred to a $25 \mathrm{~mL}$ volumetric flask and diluted with Milli-Q water.

\section{Acknowledgements}

We acknowledge financial support from the Spanish Ministry of Economy and Competitiveness (MINECO) through CTQ201232247 and CTQ2012-31335 and from the Generalitat de Catalunya through 2014 SGR 129. A.F. acknowledges the Spanish MINECO for a Ramón y Cajal Fellowship (RYC-2010-05821). J.L. is a Serra Húnter Fellow and is grateful to the Catalan Institution for Research and Advanced Studies (ICREA) Academia Program.

Keywords: conjugation · gold · hydrogels $\cdot$ metallophilicity nanoparticles

[1] H. A. Atwater, A. Polman, Nat. Mater. 2010, 9, 205-213.

[2] C. Wang, D. Astruc, Chem. Soc. Rev. 2014, 43, 7188-7216.

[3] M. G. Kang, T. Xu, H. J. Park, X. Luo, L. J. Guo, Adv. Mater. 2010, 22, $4378-4383$.

[4] L. Lu, Z. Luo, T. Xu, L. Yu, Nano Lett. 2013, 13, 59-64.

[5] T. Jägeler-Hoheisel, F. Selzer, M. Riede, K. Leo, J. Phys. Chem. C 2014, $118,15128-15135$.

[6] H. II Park, S. Lee, J. M. Lee, S. A. Nam, T. Jeon, S. W. Han, S. O. Kim, ACS Nano 2014, 8, 10305-10312.

[7] P. V. Kamat, Acc. Chem. Res. 2012, 45, 1906-1915.

[8] N. Bansal, L. X. Reynolds, A. MacLachlan, T. Lutz, R. S. Ashraf, W. Zhang, C. B. Nielsen, I. McCulloch, D. G. Rebois, T. Kirchartz, M. S. Hill, K. C. Molloy, J. Nelson, S. A. Haque, Sci. Rep. 2013, 3, 1531.

[9] Y. Ben-Shahar, F. Scotognella, N. Waiskopf, I. Kriegel, S. Dal Conte, G. Cerullo, U. Banin, Small 2015, 11, 462-471.

[10] V. K. Praveen, C. Ranjith, E. Bandini, A. Ajayaghosh, N. Armaroli, Chem Soc. Rev. 2014, 43, $4222-4242$.

[11] Y. D. Park, S. G. Lee, H. S. Lee, D. Kwak, D. H. Lee, K. Cho, J. Mater. Chem 2011, 21, 2338-2343.

[12] J. Kao, K. Thorkelsson, P. Bai, B. J. Rancatore, T. Xu, Chem. Soc. Rev. 2013 42, 2654-2678

[13] S. Ren, L.-Y. Chang, S.-K. Lim, J. Zhao, M. Smith, N. Zhao, V. Bulović, M. Bawendi, S. Gradecak, Nano Lett. 2011, 11, 3998-4002.

[14] J. Albero, P. Riente, J. N. Clifford, M. A. Pericàs, E. Palomares, J. Phys. Chem. C 2013, 117, 13374-13381.

[15] S. Lim, J. E. Song, J. A. La, E. C. Cho, Chem. Mater. 2014, 26, 3272- 3279.

[16] S. G. Jang, E. J. Kramer, C. J. Hawker, J. Am. Chem. Soc. 2011, 133, $16986-16996$.

[17] J. Zhang, C. Y. Su, Coord. Chem. Rev. 2013, 257, 1373-1408.

[18] J. C. Lima, L. Rodríguez, Inorganics 2015, 3, 1-18.

[19] H. Schmidbaur, A. Schier, Chem. Soc. Rev. 2012, 41, 370-412.

[20] A. Kishimura, T. Yamashita, T. Aida, J. Am. Chem. Soc. 2005, 127, 179183.

[21] C. A. Strassert, C. H. Chien, M. D. Galvez Lopez, D. Kourkoulos, D. Hertel, K. Meerholz, L. De Cola, Angew. Chem. Int. Ed. 2011, 50, 946-950; Angew. Chem. 2011, 123, 976-980.

[22] R. Gavara, J. Llorca, J. C. Lima, L. Rodríguez, Chem. Commun. 2013, 49, $72-74$.

[23] R. Gavara, E. Aguiló, C. Fonseca Guerra, L. Rodríguez, J. C. Lima, Inorg. Chem. 2015, 54, 5195-5203.

[24] E. Aguiló, R. Gavara, J. C. Lima, J. Llorca, L. Rodríguez, J. Mater. Chem. C 2013, 1, 5538-5547.

[25] J. Xian, Q. Hua, Z. Jiang, Y. Ma, W. Huang, Langmuir 2012, 28, 6736 6741.

[26] S. K. Ghosh, T. Pal, Chem. Rev. 2007, 107, 4797-4862.

[27] S. Srivastava, B. L. Frankamp, V. M. Rotello, Chem. Mater. 2005, 17, 487490.

[28] L. Rodríguez, M. Ferrer, R. Crehuet, J. Anglada, J. C. Lima, Inorg. Chem. 2012, 51, 7636-7641.

[29] E. J. Fernández, J. M. López-de-Luzuriaga, M. Monge, M. A. Rodríguez, O. Crespo, M. C. Gimeno, A. Laguna, P. G. Jones, Chem. Eur. J. 2000, 6, 636-644.

[30] S. Kühn, U. Håkanson, L. Rogobete, V. Sandoghdar, Phys. Rev. Lett. 2006, 97, 017402. 
[31] J. P. Rosa, J. C. Lima, P. V. Baptista, Nanotechnology 2011, 22, 415202.

[32] S. Schlücker, Angew. Chem. Int. Ed. 2014, 53, 4756-4795; Angew. Chem. 2014, 126, 4852- 4894.

[33] R. A. Álvarez-Puebla, J. Phys. Chem. Lett. 2012, 3, 857-866.

[34] M. Ferrer, M. Mounir, L. Rodríguez, O. Rosell, S. Coco, P. Gómez-Sal, A. J. Martin, J. Organomet. Chem. 2005, 690, 2200-2208.

[35] H. Yu, M. Chen, P. M. Rice, S. X. Wang, R. L. White, S. Sun, Nano Lett. 2005, 5, 379-382.

[36] C. Shen, C. Hui, T. Yang, C. Xiao, L. Bao, S. Chen, H. Ding, H. Gao, J. Tian, Chem. Mater. 2008, 20, 6939-6944.

[37] J. Park, K. An, Y. Hwang, J.-G. Park, H.-J. Noh, J.-Y. Kim, J.-H. Park, N.-M. Hwang, T. Hyeon, Nat. Mater. 2004, 3, 891-895.

[38] L. Carbone, C. Nobile, M. De Giorgi, F. Della Sala, G. Morello, P. Pompa, M. Hytch, E. Snoeck, A. Fiore, I. R. Franchini, M. Nadasan, A. F. Silvestre
L. Chiodo, S. Kudera, R. Cingolani, R. Krahne, L. Manna, Nano Lett. 2007, 7, $2942-2950$.

[39] A. G. Malyutin, R. Easterday, Y. Lozovyy, A. Spilotros, H. Cheng, O. R. Sanchez-Felix, B. D. Stein, D. G. Morgan, D. I. Svergun, B. Dragnea, L. M. Bronstein, Chem. Mater. 2015, 27, 327-335.

[40] A. Sahu, A. Khare, D. D. Deng, D. J. Norris, Chem. Commun. 2012, 48, 5458-5460.

[41] J. Yang, E. H. Sargent, S. O. Kelley, J. Y. Ying, Nat. Mater. 2009, 8, $683-$ 689.

Manuscript received: March 8, 2016

Accepted Article published: March 11, 2016

Final Article published: $\square$ U, 2016 


\section{ARTICLES}

M. Dalmases, E. Aguiló, J. Llorca,

L. Rodríguez, ${ }^{*}$ A. Figuerola*

$\mathbf{a - n}$

Exploiting Metallophilicity for the Assembly of Inorganic Nanocrystals and Conjugated Organic Molecules

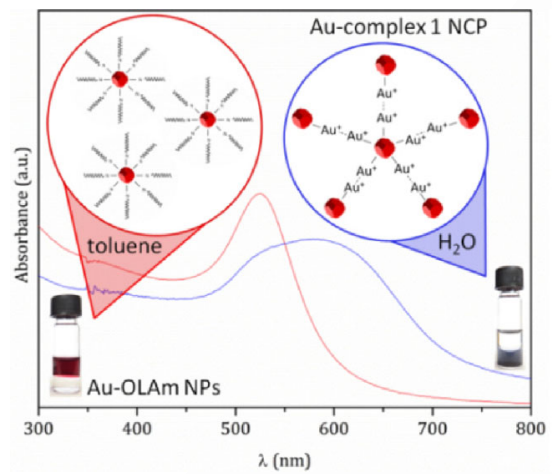

Interface design: A new source of interface interaction based on metal-metal bonds is presented. An organometallic gelator is used for the formation of hydrogel-like nanocomposites (NCPs) embedding inorganic nanoparticles and conjugated organic molecules. The establishment of metallophilic interactions at the interface between the two moieties greatly enhances the interparticle coupling, as evidenced in the case of plasmonic nanoparticles. Au-OLAm NPs: oleylamine-capped Au nanoparticles.

\#Metallophilicity for the engineering of organic-inorganic \#interfaces @UniBarcelona SPACE RESERVED FOR IMAGE AND LINK

Share your work on social media! ChemPhysChem has added Twitter as a means to promote your article. Twitter is an online microblogging service that enables its users to send and read text-based messages of up to 140 characters, known as "tweets". Please check the pre-written tweet in the galley proofs for accuracy. Should you or your institute have a Twitter account, please let us know the appropriate username (i.e., @accountname), and we will do our best to include this information in the tweet. This tweet will be posted to the journal's Twitter account @ChemPhysChem (follow us!) upon online publication of your article, and we recommended you to repost ("retweet") it to alert other researchers about your publication.

Please check that the ORCID identifiers listed below are correct. We encourage all authors to provide an ORCID identifier for each coauthor. ORCID is a registry that provides researchers with a unique digital identifier. Some funding agencies recommend or even require the inclusion of ORCID IDs in all published articles, and authors should consult their funding agency guidelines for details. Registration is easy and free; for further information, see http://orcid.org/.

Mariona Dalmases

Elisabet Aguiló

Dr. Jordi Llorca

Dr. Laura Rodríguez

Dr. Albert Figuerola http://orcid.org/0000-0003-1703-1025 